\title{
Jewel beetles (Coleoptera, Buprestidae) collected during the 2018 field survey on lejima Island, the Okinawa Islands, Ryukyus, south-western Japan
}

\author{
Yutaka Tamadera ${ }^{\ddagger}$, Hiraku Yoshitake§ \\ ‡ Laboratory of Entomology, Tokyo University of Agriculture, Atsugi, Japan \\ $\S$ Kyushu Okinawa Agricultural Research Center (Itoman residence), NARO, Itoman, Japan
}

Corresponding author: Yutaka Tamadera (iltamamusi@gmail.com)

Academic editor: Paolo A. Audisio

Received: 26 Nov 2019 |Accepted: 20 Jan 2020 | Published: 31 Jan 2020

Citation: Tamadera Y, Yoshitake H (2020) Jewel beetles (Coleoptera, Buprestidae) collected during the 2018 field survey on lejima Island, the Okinawa Islands, Ryukyus, south-western Japan. Biodiversity Data Journal 8: e48785. https://doi.org/10.3897/BDJ.8.e48785

\begin{abstract}
Background

Only one jewel beetle, Chrysodema (Marcsikiella) dalmanni (Eschscholtz) (Coleoptera, Buprestidae) has hitherto been recorded from lejima Is. in the Okinawa Isls., Ryukyus, south-western Japan.
\end{abstract}

\section{New information}

A total of seven jewel beetles were collected on lejima Is. The following six species are newly recorded from the island: 1) Paratrachys princeps chujoi Kurosawa, 2) Chrysodema (Chrysodema) lewisii Saunders, 3) Chalcophora japonica oshimana Schönfeldt, 4) Coraebus hastanus Gory and Laporte, 5) Sambus quadricolor quadricolor Saunders and 6) Agrilus okinawensis shiozakii Tôyama. 


\section{Keywords}

Buprestid beetle, fauna, distribution, new record

\section{Introduction}

The Okinawa Islands in the Ryukyus, south-western Japan (Fig. 1) has one of the highest species diversity of jewel beetles (Coleoptera, Buprestidae) in Japan. From the islands, 44 buprestid species belonging to 18 genera in four subfamilies have hitherto been recorded, including five endemic species and five endemic subspecies (Ohmomo and Fukutomi 2013, Hattori 2014). This species number accounts for about $20 \%$ of all Japanese buprestid beetles. The buprestid fauna of the Okinawa Isls. has hitherto been well surveyed particularly in the largest island, Okinawajima Is. (area ca. 1,207 km²; 44 known species), while our knowledge on the fauna of the other islands is still limited due to the lack of sufficient surveys. For example, only eight species had been recorded from the second largest island, Kumejima Is. (area ca. $60 \mathrm{~km}^{2}$ ) until recently, when five more species were recorded by Tamadera et al. (2019).

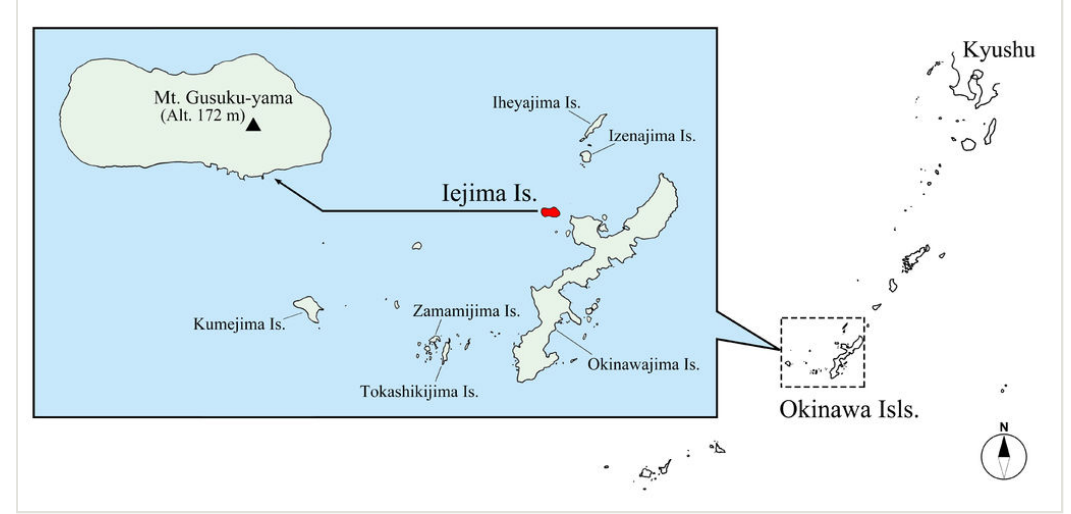

Figure 1. doi

Location of the Okinawa Islands and lejima Island in the Ryukyus, south-western Japan.

lejima Island, about $9 \mathrm{~km}$ off the north-western coast of the Motobu Peninsula in Okinawajima Is., is the third largest island (area ca. $22.8 \mathrm{~km}^{2}$ ) in the Okinawa Isls. (Fig. 1). lejima Is. is mostly flat and covered with cropland, except a small evergreen broad-leaved forest is spreading in Mt. Gusuku-yama (altitude ca. $172 \mathrm{~m}$ ), located in the central east (Figs 1, 2). Presently, only one buprestid beetle, Chrysodema (Marcsikiella) dalmanni (Eschscholtz, 1837), is known from the island (Kohama 2015, Yao 2016). However, more buprestids are expected to occur on the island, since no investigations on the buprestid fauna have been conducted until now. In June 2018, we conducted a field survey on lejima Is. and collected seven buprestid beetles, six of which are new to the fauna of the island. In this paper, we will report them for the first time from lejima Is. 


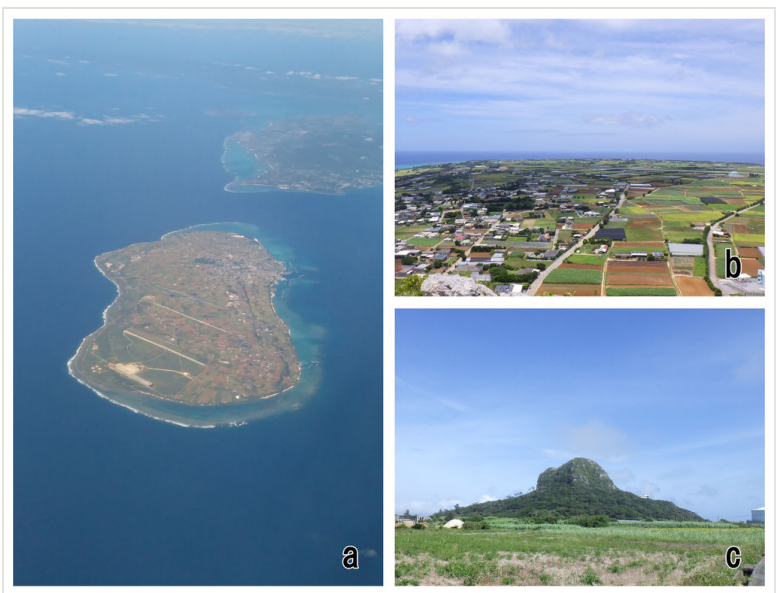

Figure 2. doi

lejima Island: a, Aerial view; b, View from the top of Mt. Gusuku-yama; c, Mt. Gusuku-yama.

\section{Materials and methods}

Specimens used in this study were collected on lejima Is. by the authors in a period from 8 th to $11^{\text {th }}$ June 2018 . All the specimens were collected by net-sweeping or beating of plants, as well as by visual searching and then examined and identified by the first author (YT) under an Olympus SHZ10 stereomicroscope. In addition, associated plants with each buprestid species were identified by him. Scientific names of buprestid beetles followed recent catalogues edited by Löbl and Löbl (2016) and plant nomenclature followed Yonekura and Kajita (2003). Finally, all the specimens are preserved in the private collection of the first author.

A habitus photograph of each species was taken under a Canon 8000D digital camera. Each final image was assembled from a series of photographs with different focus planes, using the computer freeware CombineZP (Hadley 2010).

Abbreviations used for collector names in this study are as follows: HY - Hiraku Yoshitake; YT - Yutaka Tamadera.

\section{Taxon treatments}

Paratrachys princeps chujoi Kurosawa, 1976

\section{Material}

a. islandGroup: Ryukyus; island: lejima Island; country: Japan; stateProvince: Okinawa; locality: Wajii-tenbôdai, Higashie-ue, le-son; eventDate: 06/09/2018; individualCount: 3; sex: unknown; lifeStage: adult; recordedBy: Yutaka Tamadera; identifiedBy: Yutaka Tamadera; dateldentified: 2018; basisOfRecord: PreservedSpecimen 


\section{Distribution}

Japan: the Okinawa Isls. (Okinawajima Is.; lejima Is. - new record) and Yaeyama Isls. (Ishigakijima Is.) (Ohmomo and Fukutomi 2013).

\section{Notes}

New to lejima Is., these specimens were collected by visual searching and beating Ficus pumila (Moraceae) which grows on a cliff facing the sea (Figs 3d, 4a).
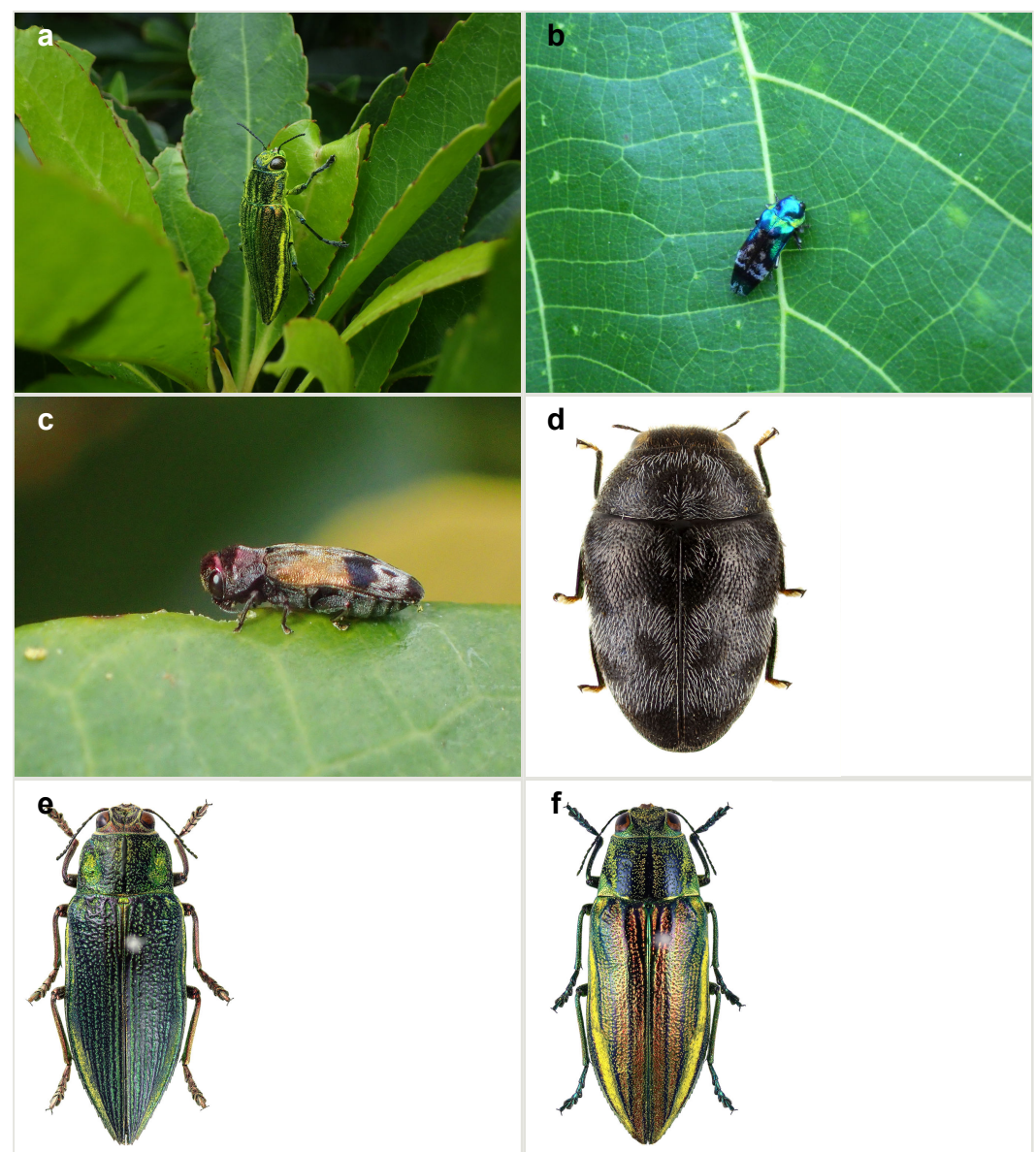

Figure 3.

Buprestid beetles from lejima Island.

a: Chrysodema (Chrysodema) lewisii on Elaeocarpus zollingeri. doi

b: Coraebus hastanus on Macaranga tanarius. doi

c: Sambus quadricolor quadricolor on Ficus pumila. doi

d: Paratrachys princeps chujoi. doi

e: Chrysodema (Marcsikiella) dalmanni. doi

f: Chrysodema (Chrysodema) lewisii. doi 

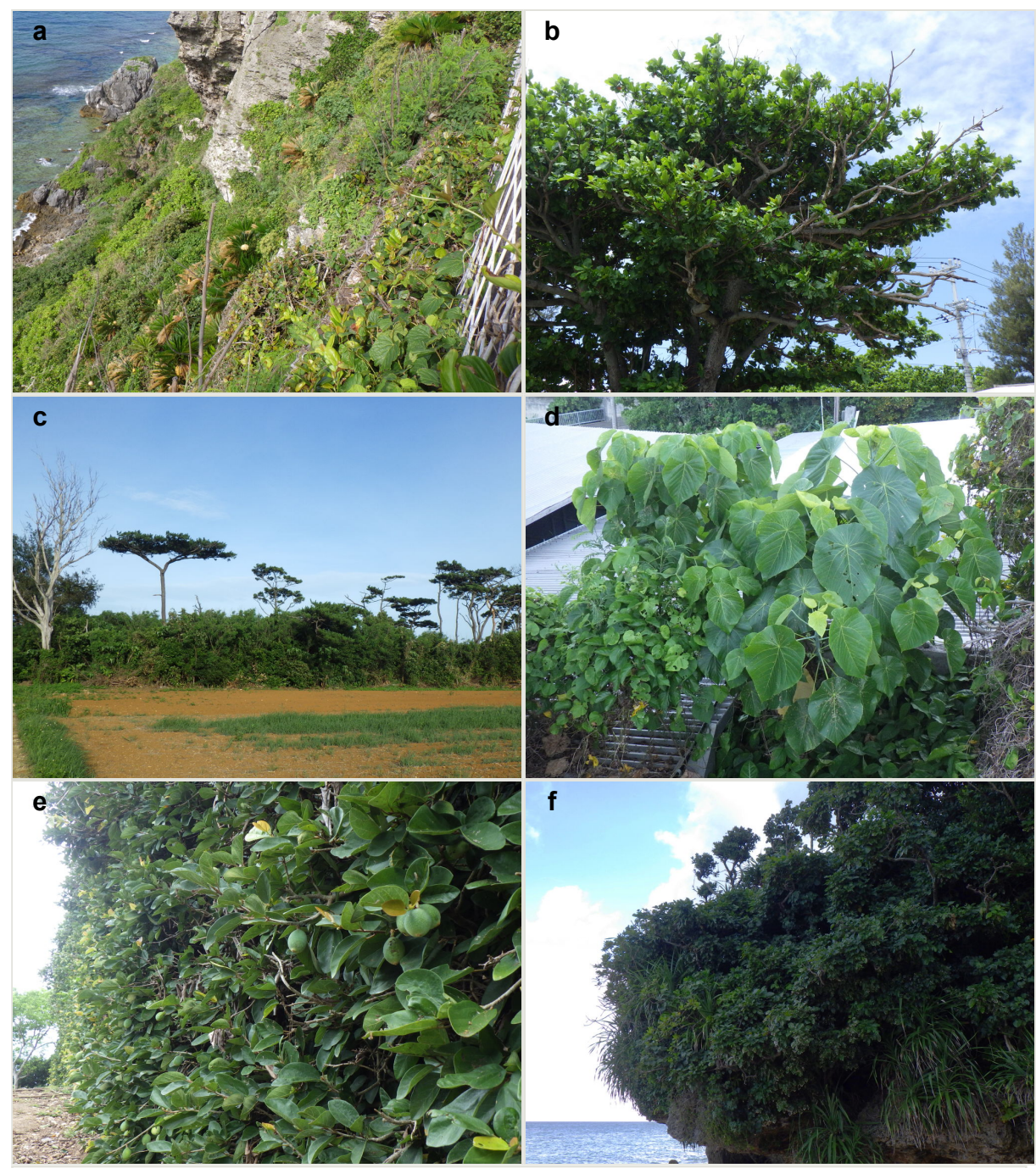

Figure 4.

Habitats and host plants of buprestid beetles.
a: A cliff facing the sea. doi
b: Terminalia catappa infested by Chrysodema (Marcsikiella) dalmanni. doi
c: A small forest left in cropland. doi
d: Macaranga tanarius infested by adult Coraebus hastanus. doi
e: Ficus pumila on an artificial wall. doi
f: Pongamia pinnata in a coastal forest. doi 


\section{Chrysodema (Marcsikiella) dalmanni (Eschscholtz, 1837)}

\section{Materials}

a. islandGroup: Ryukyus; island: lejima Island; country: Japan; stateProvince: Okinawa; locality: Ishara-hama, Higashie-mae, le-son; eventDate: 2018-06-10; individualCount: 14; sex: 10 males, 4 females; lifeStage: adult; recordedBy: Yutaka Tamadera; identifiedBy: Yutaka Tamadera; dateldentified: 2018; basisOfRecord: PreservedSpecimen

b. $\quad$ islandGroup: Ryukyus; island: lejima Island; country: Japan; stateProvince: Okinawa; locality: Ishara-hama, Higashie-mae, le-son; eventDate: 2018-06-11; individualCount: 6; sex: 5 males, 1 female; lifeStage: adult; recordedBy: Yutaka Tamadera; identifiedBy: Yutaka Tamadera; dateldentified: 2018; basisOfRecord: PreservedSpecimen

c. islandGroup: Ryukyus; island: lejima Island; country: Japan; stateProvince: Okinawa; locality: Ishara-hama, Higashie-mae, le-son; eventDate: 2018-06-11; individualCount: 6; sex: 4 males, 2 females; lifeStage: adult; recordedBy: Hiraku Yoshitake; identifiedBy: Yutaka Tamadera; dateldentified: 2018; basisOfRecord: PreservedSpecimen

\section{Distribution}

Japan: the Amami Isls. (Amami-Ôshima Is., Kikaijima Is. and Tokunoshima Is.), Okinawa Isls. (Iheyajima Is., Nohojima Is., Okinawajima Is., Hamahigajima Is., Zamamijima Is., Tokashikijima Is., Akajima Is., Fukajishima Is. andKumejima Is.; Iejima Is. - new record), Miyako Isls. (Miyakojima Is.) and Yaeyama Isls. (Ishigakijima Is., Iriomotejima Is., Kohamajima Is., Kuroshima Is., Haterumajima Is. and Yonagunijima Is.); Taiwan; Philippines; and Indonesia (Ohmomo and Fukutomi 2013, Aoki 2014, Kohama 2015, Inada et al. 2015, Yao 2016, Inada 2016, Sakai 2016b, Kusui and Miyagi 2017, Hinoki 2018, Tamadera et al. 2019).

\section{Notes}

These specimens were collected by net-sweeping Terminalia catappa (Combretaceae) (Figs $3 e, 4 b$ ). In the collecting site, some adult exit holes of $C$. (M.) dalmanni were observed in thick dead branches of the plant.

\section{Chrysodema (Chrysodema) lewisii Saunders, 1873}

\section{Materials}

a. islandGroup: Ryukyus; island: lejima Island; country: Japan; stateProvince: Okinawa; locality: Mt. Gusuku-yama, Higashie-ue, le-son; eventDate: 2018-06-08; individualCount: 3; sex: 3 males; lifeStage: adult; recordedBy: Yutaka Tamadera; identifiedBy: Yutaka Tamadera; dateldentified: 2018; basisOfRecord: PreservedSpecimen

b. $\quad$ islandGroup: Ryukyus; island: lejima Island; country: Japan; stateProvince: Okinawa; locality: Mt. Gusuku-yama, Higashie-ue, le-son; eventDate: 2018-06-09; individualCount: 9; sex: 5 males, 4 females; lifeStage: adult; recordedBy: Yutaka Tamadera; identifiedBy: Yutaka Tamadera; dateldentified: 2018; basisOfRecord: PreservedSpecimen

c. islandGroup: Ryukyus; island: lejima Island; country: Japan; stateProvince: Okinawa; locality: Mt. Gusuku-yama, Higashie-ue, le-son; eventDate: 2018-06-10; individualCount: 
1; sex: female; lifeStage: adult; recordedBy: Yutaka Tamadera; identifiedBy: Yutaka Tamadera; dateldentified: 2018; basisOfRecord: PreservedSpecimen

d. islandGroup: Ryukyus; island: lejima Island; country: Japan; stateProvince: Okinawa; locality: Mt. Gusuku-yama, Higashie-ue, le-son; eventDate: 2018-06-11; individualCount: 6; sex: 3 males, 3 females; lifeStage: adult; recordedBy: Yutaka Tamadera; identifiedBy: Yutaka Tamadera; dateldentified: 2018; basisOfRecord: PreservedSpecimen

\section{Distribution}

Japan: Honshu, Shikoku, Kyushu, the Izu Isls. (Hachijôjima Is.), Tsushima Is., Kuroshima Is. (in Mishima-mura), Ôsumi Isls. (Tanegashima Is., Yakushima Is. and Kuchinoerabujima Is.), Tokara Isls. (Nakanoshima Is. and Akusekijima Is.), Amami Isls. (Amami-Ôshima Is., Yorojima Is., Tokunoshima Is. and Okinoerabujima Is.), Okinawa IsIs. (Iheyajima Is., Okinawajima Is., Tokashikijima Is., Zamamijima Is., Akajima Is., Gerumajima Is. and Kumejima Is.; lejima Is. - new record), Miyako Isls. (Miyakojima Is.) and Yaeyama Isls. (Ishigakijima Is., Iriomotejima Is., Kuroshima Is. and Yonagunijima Is.); and Taiwan (Takai 1991, Ohmomo and Fukutomi 2013, Inada et al. 2015, Sakai 2016a, Sakai 2016b, Hinoki 2018).

\section{Notes}

New to lejima Is., these specimens were collected by visual searching and netsweeping Elaeocarpus zollingeri (Elaeocarpaceae) and Toxicodendron succedaneum (Anacardiaceae) (Fig. 3a, f). Toxicodendron succedaneum is not recorded as a host plant of $C$. (C.) lewisii, but this species may be associated with the plant at least during the adult stage, because some specimens were collected from it at several places.

\section{Chalcophora japonica oshimana Schönfeldt, 1890}

\section{Materials}

a. islandGroup: Ryukyus; island: lejima Island; country: Japan; stateProvince: Okinawa; locality: near Beigun-hojo-hikôjô-atochi, Nishie-ue, le-son; eventDate: 06/10/2018; individualCount: 2; sex: 1 male, 1 female; lifeStage: adult; recordedBy: Yutaka Tamadera; identifiedBy: Yutaka Tamadera; dateldentified: 2018; basisOfRecord: PreservedSpecimen

b. $\quad$ islandGroup: Ryukyus; island: lejima Island; country: Japan; stateProvince: Okinawa; locality: near Beigun-hojo-hikôjô-atochi, Nishie-ue, le-son; eventDate: 06/11/2018; individualCount: 2; sex: 1 male, 1 female; lifeStage: adult; recordedBy: Yutaka Tamadera; identifiedBy: Yutaka Tamadera; dateldentified: 2018; basisOfRecord: PreservedSpecimen

\section{Distribution}

Japan: the Amami Isls. (Amami-Ôshima Is., Kikaijima Is., Kakeromajima Is., Yorojima Is., Tokunoshima Is. and Okinoerabujima Is.), Okinawa Isls. (Iheyajima Is., Okinawajima Is., Agunijima Is., Zamamijima Is., Akajima Is., Gerumajima Is. and Fukajishima Is.; lejima Is. - new record) and Miyako Isls. (Miyakojima Is.) (Ohmomo and Fukutomi 2013, Inada et al. 2015, Inada 2016, Sakai 2016b, Sakai 2017a). 


\section{Notes}

New to lejima Is., these specimens were collected by net-sweeping Pinus luchuensis (Pinaceae). The collecting site is a small forest left in cropland (Figs 4c, 5a).

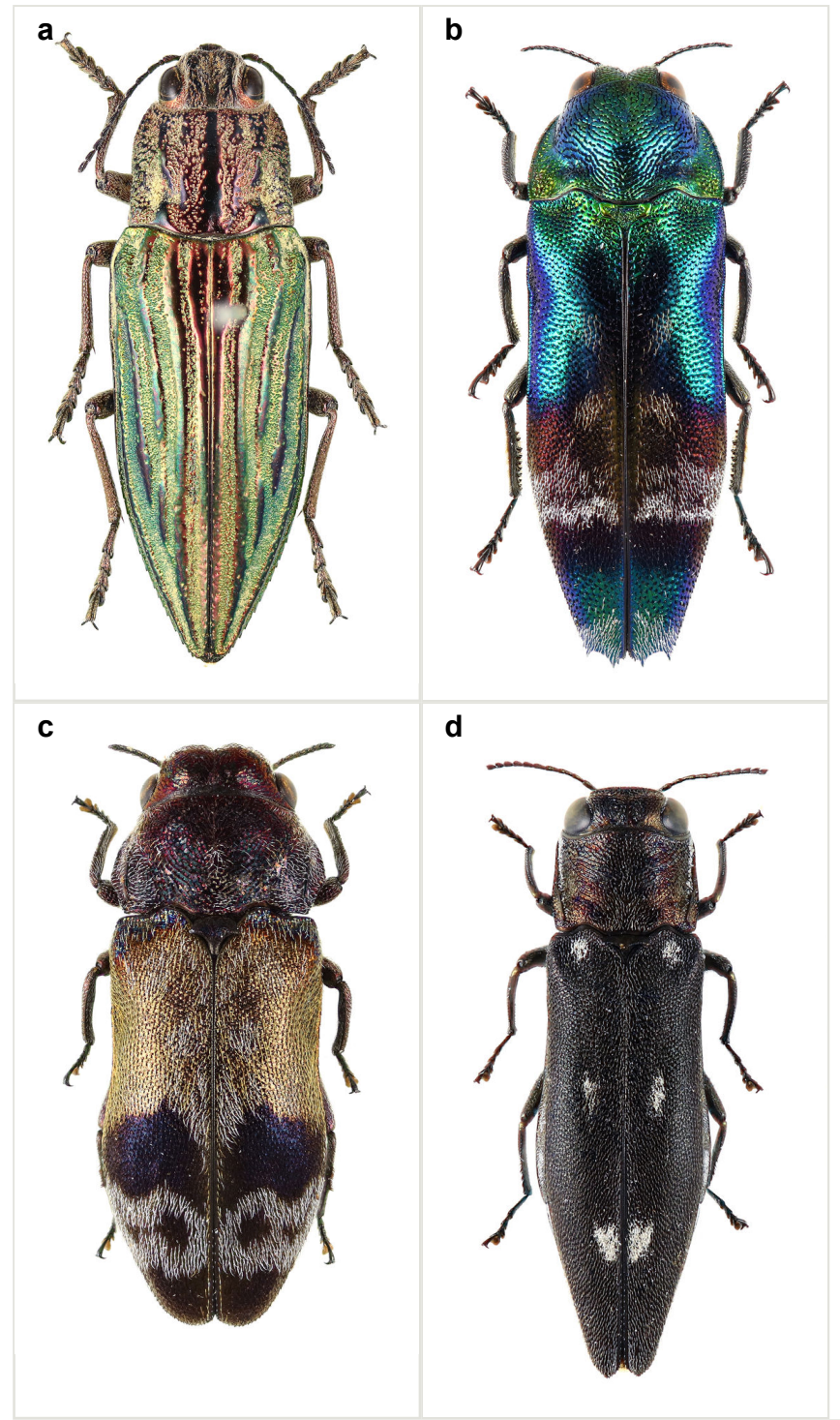

Figure 5.

Buprestid beetles from lejima Island.

a: Chalcophora japonica oshimana. doi

b: Coraebus hastanus. doi

c: Sambus quadricolor quadricolor. doi

d: Agrilus okinawensis shiozakii. doi 


\section{Coraebus hastanus Gory and Laporte, 1839}

\section{Materials}

a. $\quad$ islandGroup: Ryukyus; island: lejima Island; country: Japan; stateProvince: Okinawa; locality: Mt. Gusuku-yama, Higashie-ue, le-son; eventDate: 2018-06-09; individualCount: 2; sex: 2 males; lifeStage: adult; recordedBy: Yutaka Tamadera; identifiedBy: Yutaka Tamadera; dateldentified: 2018; basisOfRecord: PreservedSpecimen

b. $\quad$ islandGroup: Ryukyus; island: lejima Island; country: Japan; stateProvince: Okinawa; locality: near Shôtaiji Temple, Nishie-mae, le-son; eventDate: 2018-06-10; individualCount: 3; sex: 1 male, 2 females; lifeStage: adult; recordedBy: Yutaka Tamadera; identifiedBy: Yutaka Tamadera; dateldentified: 2018; basisOfRecord: PreservedSpecimen

c. islandGroup: Ryukyus; island: lejima Island; country: Japan; stateProvince: Okinawa; locality: near Kawahira-kôminkan, Kawahira, le-son; eventDate: 2018-06-10; individualCount: 4; sex: 3 males, 1 female; lifeStage: adult; recordedBy: Yutaka Tamadera; identifiedBy: Yutaka Tamadera; dateldentified: 2018; basisOfRecord: PreservedSpecimen

\section{Distribution}

Japan: the Amami Isls. (Amami-Ôshima Is., Kikaijima Is., Tokunoshima Is. and Okinoerabujima Is.), Okinawa Isls. (Iheyajima Is., Izenajima Is., Okinawajima Is., Senagajima Is., Hamahigajima Is., Miyagijima Is., Tsukenjima Is., Yabuchijima Is. and Kumejima Is.; lejima Is. — new record), Miyako Isls. (Miyakojima Is. and Ikemajima Is.) and Yaeyama Isls. (Ishigakijima Is., Iriomotejima Is. and Kuroshima Is.); Taiwan; China; Nepal; Bhutan; India; Burma; Thailand; Laos; Vietnam; Indonesia; and Philippines (Xu et al. 2013, Ohmomo and Fukutomi 2013, Kusui 2017, Kusui and Miyagi 2017, Sakai 2017b, Hinoki 2018, Tamadera and Yoshitake 2018, Tamadera et al. 2018, Tamadera and Shigetoh 2019).

\section{Notes}

New to lejima Is., these specimens were collected by visual searching and netsweeping Macaranga tanarius (Euphorbiaceae) which is not recorded as a host plant of C. hastanus (Figs $3 b, 4 d, 5 b$ ). This buprestid beetle is almost certainly associated with M. tanarius, because all specimens were collected only from the plant. In addition, Mallotus japonicus (Euphorbiaceae), which has been known as a host plant of this species, was not found in and around each collecting site, as far as we searched.

\section{Sambus quadricolor quadricolor Saunders, 1873}

\section{Materials}

a. islandGroup: Ryukyus; island: lejima Island; country: Japan; stateProvince: Okinawa; locality: Mt. Gusuku-yama, Higashie-ue, le-son; eventDate: 06/08/2018; individualCount: 43; sex: 25 males, 18 females; lifeStage: adult; recordedBy: Yutaka Tamadera; identifiedBy: Yutaka Tamadera; dateldentified: 2018; basisOfRecord: PreservedSpecimen 
b. islandGroup: Ryukyus; island: lejima Island; country: Japan; stateProvince: Okinawa; locality: Mt. Gusuku-yama, Higashie-ue, le-son; eventDate: 06/08/2018; individualCount: 24; sex: 9 males, 15 females; lifeStage: adult; recordedBy: Hiraku Yoshitake; identifiedBy: Yutaka Tamadera; dateldentified: 2018; basisOfRecord: PreservedSpecimen

c. islandGroup: Ryukyus; island: lejima Island; country: Japan; stateProvince: Okinawa; locality: Mt. Gusuku-yama, Higashie-ue, le-son; eventDate: 06/09/2018; individualCount: 7; sex: 4 males, 3 females; lifeStage: adult; recordedBy: Yutaka Tamadera; identifiedBy: Yutaka Tamadera; dateldentified: 2018; basisOfRecord: PreservedSpecimen

\section{Distribution}

Japan: Honshu (Kanagawa Pref. and Shizuoka Pref.), Kyushu, Sarushima Is. (in Yokosuka-shi), the Ôsumi Isls. (Yakushima Is.), Amami Isls. (Amami-Ôshima Is.) and Okinawa Isls. (Okinawajima Is. and Hamahigajima Is.; lejima Is. - new record) (Ohmomo and Fukutomi 2013, Kusui and Miyagi 2017).

\section{Notes}

New to lejima Is., these specimens were collected by visual searching, net-sweeping and beating Ficus pumila which grows on an artificial wall (Figs 3c, 4e, 5c).

\section{Agrilus okinawensis shiozakii Tôyama, 1985}

\section{Materials}

a. islandGroup: Ryukyus; island: lejima Island; country: Japan; stateProvince: Okinawa; locality: GI beach, Kawahira, le-son; eventDate: 06/09/2018; individualCount: 2; sex: 1 male, 1 female; lifeStage: adult; recordedBy: Yutaka Tamadera; identifiedBy: Yutaka Tamadera; dateldentified: 2018; basisOfRecord: PreservedSpecimen

b. $\quad$ islandGroup: Ryukyus; island: lejima Island; country: Japan; stateProvince: Okinawa; locality: near GI beach, Nishie-mae, le-son; eventDate: 06/09/2018; individualCount: 6; sex: 3 males, 3 females; lifeStage: adult; recordedBy: Yutaka Tamadera; identifiedBy: Yutaka Tamadera; dateldentified: 2018; basisOfRecord: PreservedSpecimen

c. islandGroup: Ryukyus; island: lejima Island; country: Japan; stateProvince: Okinawa; locality: near Shôtaiji Temple, Nishie-mae, le-son; eventDate: 06/10/2018; individualCount: 6; sex: 1 male, 5 females; lifeStage: adult; recordedBy: Yutaka Tamadera; identifiedBy: Yutaka Tamadera; dateldentified: 2018; basisOfRecord: PreservedSpecimen

\section{Distribution}

Japan: the Amami Isls. (Amami-Ôshima Is.) and Okinawa Isls. (Okinawajima Is., Ikeijima Is. and Kumejima Is.; lejima Is. - new record) (Ohmomo and Fukutomi 2013, Tamadera et al. 2019).

\section{Notes}

New to lejima Is., these specimens were collected by net-sweeping Pongamia pinnata (Fabaceae) (Figs 4f, 5d). 


\section{Discussion}

By this study, the number of buprestid beetles, known from lejima Is., has been increased from one to seven species belonging to six genera in three subfamilies. Amongst them, four species, Chrysodema (Marcsikiella) dalmanni, Chrysodema (Chrysodema) lewisii, Chalcophora japonica oshimana and Coraebus hastanus, are common species and broadly distributed in the Okinawa Isls. (Table 1), since their associated plants are also common and abundant in many islands. With regard to Chalcophora japonica oshimana, marine drifts might have played a role in the dispersal of the species from island to island in the Okinawa Isls. (Kurosawa 1974). On the other hand, the remaining three species, Paratrachys princeps chujoi, Sambus quadricolor quadricolor and Agrilus okinawensis shiozakii, are relatively rare and known from only a few islands of the Okinawa Isls. (Table 1), but their associated plants are never rare and broadly distributed. Thus, these buprestids are likely to be distributed in the other adjacent islands.

\section{Table 1.}

Commonality of buprestid species between lejima Island and some other islands of the Okinawa Islands (area $\left(\mathrm{km}^{2}\right)$ of and the number of known species from each island are indicated in parentheses).

\begin{tabular}{|c|c|c|c|c|c|c|}
\hline \multirow[t]{2}{*}{ Species } & \multicolumn{6}{|c|}{ Islands } \\
\hline & $\begin{array}{l}\text { lejima } \\
(22.8 ; 7)\end{array}$ & $\begin{array}{l}\text { Okinawajima } \\
(1,207 ; 44)\end{array}$ & $\begin{array}{l}\text { Kumejima } \\
(59.5 ; 13)\end{array}$ & $\begin{array}{l}\text { Iheyajima } \\
(20.7 ; 8)\end{array}$ & $\begin{array}{l}\text { Tokashikijima } \\
(15.3 ; 8)\end{array}$ & $\begin{array}{l}\text { Zamamijima } \\
(6.7 ; 5)\end{array}$ \\
\hline Paratrachys princeps chujoi & + & + & - & - & - & - \\
\hline Chrysodema (Marcsikiella) dalmanni & + & + & + & + & + & + \\
\hline Chrysodema (Chrysodema) lewisii & + & + & + & + & + & + \\
\hline Chalcophora japonica oshimana & + & + & + & + & + & + \\
\hline Coraebus hastanus & + & + & + & + & - & - \\
\hline Sambus quadricolor quadricolor & + & + & - & - & - & - \\
\hline Agrilus okinawensis shiozakii & + & + & + & - & - & - \\
\hline
\end{tabular}

Generally, larger islands tend to have more species, while smaller islands tend to have fewer species (Whittaker and Fernández-Palacios 2007), but the number of buprestid species presently known from lejima Is. is less than that from some smaller islands in the Okinawa Isls. (Table 1). Probably due to excessive deforestation in lejima Is., the buprestid fauna of this island seems to have become considerably poorer than in the past. However, it is still possible that some additional buprestids will be found on lejima Is., since our field survey was conducted during a short period in the early summer of 2018. 


\section{References}

- $\quad$ Aoki I (2014) [Chrysodema dalmanni collected from Haterumajima Island]. GekkanMushi (526): 22-23. [In Japanese].

- Hadley A (2010) CombineZP. http://www.hadleyweb.pwp.blueyonder.co.uk.

- Hattori T (2014) New species of the genus Toxoscelus (Insecta, Coleoptera, Buprestidae) from Taiwan and the Ryukyu Islands. Bulletin of the National Science Museum, Tokyo, Series A, Zoology, Supplement 8: 1-35.

- Hinoki T (2018) [Buprestid beetles collected from Kuroshima and Kohamajima Islands in the Yaeyama Islands]. Gekkan-Mushi (568): 55. [In Japanese].

- Inada S, Yamada W, Fukutomi H (2015) [Buprestid beetles of the Kerama Islands]. Gekkan-Mushi (537): 4-9. [In Japanese].

- Inada S (2016) [Records of buprestid beetles from Iheyajima and Agunijima Islands (Okinawa Prefecture)]. Gekkan-Mushi (550): 8. [In Japanese].

- Kohama T (2015) [Records of Chrysodema dalmanni from lejima Island, with observations of its adult on Planchonella obovata]. Pulex (94): 676. [In Japanese].

- Kurosawa Y (1974) A revision of the East Asian species of the genus Chalcophora (Coleoptera, Buprestidae), with special reference to their distribution and differentiation. Memoires of the National Science Museum, Tokyo 7: 169-192.

- Kusui Y (2017) [A list of Coleoptera of Senagajima Island (Tomigusuku-shi, Okinawa Prefecture) (from 1987 to 2007)]. Insects of Loochoos (41): 25-32. [In Japanese].

- Kusui Y, Miyagi A (2017) [A list of Coleoptera of Hamahigajima Island (Uruma-shi)]. Insects of Loochoos (41): 39-49. [In Japanese].

- Löbl I, Löbl D (2016) Catalogue of Palaearctic Coleoptera 3. Scarabaeoidea, Scirtoidea, Dascilloidea, Buprestoidea and Byrrhoidea. Revised and updated edition. Brill, Leiden \& Boston, $983 \mathrm{pp}$.

- Ohmomo S, Fukutomi H (2013) The Buprestid Beetles of Japan. Mushi-Sha's Iconographic Series of Insects. 7. Mushi-sha, Tokyo, 206 pp. [In Japanese, with English book title and summary].

- $\quad$ Sakai S (2016a) [New records of Chrysodema lewisii from Tokunoshima Island]. Gekkan-Mushi (548): 55. [In Japanese].

- Sakai S (2016b) [Records of four buprestid beetles from Iheyajima and Nohojima Islands]. Gekkan-Mushi (550): 9. [In Japanese].

- Sakai S (2017a) [Records of two buprestid beetles from Kakeromajima and Yorojima Islands]. Gekkan-Mushi (559): 57. [In Japanese].

- Sakai S (2017b) [Records of Coraebus hastanus oberthueri from Kumejima Island]. Gekkan-Mushi (562): 27. [In Japanese].

- $\quad$ Takai Y (1991) [The 1981 biological survey in Okinoerabujima Island]. Leben (21): 31-45. [In Japanese].

- Tamadera Y, Yoshitake H (2018) Buprestid beetles collected on Tokunoshima Island, the Ryukyus, southwest Japan. Gekkan-Mushi (565): 12-17. [In Japanese, with English title and summary].

- $\quad$ Tamadera Y, Nagano H, Kojima H (2018) [Records of two buprestid beetles from Izenajima Island in the Okinawa Islands]. Sayabane, New Series (32): 49-50. [In Japanese]. 
- $\quad$ Tamadera Y, Shigetoh H (2019) [Records of four buprestid beetles from the islands (Ikeijima, Miyagijima, Yabuchijima and Tsukenjima Islands) in Uruma-shi, Okinawa Prefecture. Sayabane, New Series (36): 19-20. [In Japanese].

- $\quad$ Tamadera Y, Yoshitake H, Nakamura R (2019) Buprestid beetles collected from Kumejima Island in the Okinawa Islands, Ryukyus, southwest Japan. Sayabane, New Series (33): 39-43. [In Japanese, with English title and summary].

- Whittaker RJ, Fernández-Palacios JM (2007) Island Biogeography: Ecology, Evolution, and Conservation. Second edition. Oxford University Press, New York, 401 pp.

- Xu H, Kubáň V, Volkovitsh MG, Ge S, Bai M, Yang X (2013) Morphological variability and taxonomy of Coraebus hastanus Gory \& Laporte de Castelnau, 1839 (Coleoptera: Buprestidae: Agrilinae: Coraebini: Coraebina). Zootaxa 3682 (1): 178-190. https:// doi.org/10.11646/zootaxa.3682.1.9

- Yao A (2016) [Records of Chrysodema dalmanni from lejima Island, Okinawa Prefecture]. Gekkan-Mushi (540): 54-55. [In Japanese].

- Yonekura K, Kajita T (2003) BG Plants (Ylist) [online]. http://ylist.info. Accessed on: 2019-11-07. 